\title{
Curbside Consultation in Cornea and External Disease: 49 Clinical Questions Editors: Francis W. Price Jr., Marianne O. Price, Erik Letko, 2010, 272 pp. , Softcover ISBN: 978-1-55642-931-6 Slack Inc
}

\author{
Daniel Kook • Patricia Kook
}

Received: 24 July 2012 / Accepted: 27 July 2012 /Published online: 15 September 2012

(C) Springer-Verlag 2012

The book "Curbside Consultation in Cornea and External Disease" is part of the "Curbside Consultation Series" that has been designed to provide ophthalmologists with practical, evidence-based answers to questions asked during informal consultations between colleagues. The concept of this series of books published by Slack Inc. is to provide medical information through the convenience of a conversation. Experts that are leaders in their specific field answer questions that pertain to ophthalmologists' daily clinical routines, and provide the reader with advice, their preferences and a personal opinion.

The editor of this 272-page English-language book is Francis W. Price Jr.; associate editors Marianne Price and Erik Letko join him. This well-structured book comprises 49 chapters organized into six sections, in the unique format of clinical questions and their answers (Q\&A). The sections are "Corneal Degeneration and Dystrophy"(nine Q\&A), "External Eye Disease and Tumors"(17 Q\&A), "Infection"(15 Q\&A),"Trauma”(four Q\&A) and finally "Postoperative"(four Q\&A). Each chapter is written by a renowned

D. Kook $(\bowtie)$

Department of Ophthalmology, Ludwig-Maximilians-University Munich,

Mathildenstrasse 8,

80366 Munich, Germany

e-mail: daniel.kook@med.uni-muenchen.de

P. Kook

Department of Ophthalmology, Technical University of Munich, Ismaninger Strasse 22,

81675 Munich, Germany

e-mail: patricia.kook@mri.tum.de corneal specialist, and the variety of nationalities represented reflects the international character of the book. Due to the plentitude of individual authors and their co-authors, there is broad variation in the structure of each chapter, which one may find either inhomogeneous and less than systematic, or rather refreshing due to the question and answer concept. Of outstanding interest in this context is one book chapter, in which three experts from different countries (USA, Europe, India) explain their individual approach to working up and treating a patient with a corneal ulcer. Numerous good quality, full colour photographic images, diagrams, and references accompany the text, serving to enhance and illustrate clinical diagnoses and treatment plans.

The information provided in the book is geared to the ophthalmology resident, the general ophthalmologist, and the experienced corneal and refractive surgeon. The book focuses on practical tips regarding the management of a specific disease, and less on its epidemiology and pathophysiology. From the reviewers' differing perspectives of corneal and refractive surgeon and ophthalmology resident in her final year, the book is well updated and does indeed target the intended readership. It provides both basic and specific information in every chapter, e.g. demonstration of potential pitfalls in the diagnostic work-up, detailed postoperative treatment regimens, and valuable treatment adjustments. The question and individual answer style makes the book convenient to read and the information easy to digest.

In summary we can highly recommend the book to anyone involved in the diagnostic work-up and treatment of common and complex corneal and external diseases, who is looking for practical guidelines in her/his clinical routine. 